\title{
PIMENTAS DO GÊNERO Capsicum CULTIVADAS EM RORAIMA, AMAZÔNIA BRASILEIRA. I. ESPÉCIES DOMESTICADAS
}

\author{
Reinaldo Imbrozio BARBOSA', Francisco Joaci Freitas LUZ ${ }^{2}$, Herundino \\ Ribeiro do NASCIMENTO FILHO ${ }^{3}$, Cice Batalha MADURO
}

\begin{abstract}
RESUMO - Foram inventariadas as pimentas domesticadas do gênero Capsicum que são cultivadas no Estado de Roraima, extremo norte da Amazônia brasileira. O levantamento foi realizado em comunidades indígenas e não indígenas. Dos 163 acessos registrados, C. chinense Jacq. (76,7\%) foi a espécie com o maior número, seguida de $C$. frutescens L. $(9,8 \%$ ), C. annuиm L. $(8,0 \%)$ e C. baccatum v. pendulum Wild. $(5,5 \%)$. As formas de fruto mais encontradas foram "alongada" $(42,9 \%)$ e "ovalada" $(27,0 \%)$. C. chinense apresentou a maior diversidade de formas enquanto que as demais estavam concentradas na forma "alongada". A cor predominante dos frutos maduros foi a vermelha $(64,4 \%)$. Isoladamente, $C$. chinense foi melhor distribuída entre as cores básicas amarela $(44,8 \%)$ e vermelha $(55,2 \%)$, independente das diferentes tonalidades assumidas por cada acesso (alaranjado, vermelho-escuro, etc). O nível de pungência sensorial com maior número de registros foi o "alto" (62,6\%), seguido do "médio" (16,0\%), "baixo" (15,3\%) e "muito alto" (6,1\%). Dos 105 acessos de coloração vermelha, 67,6\% possuía pungência "alta" ou "muito alta". C. chinense do tipo "murupi" e "olho-de-peixe", juntamente com "malagueta" ( $C$. frutescens) são os morfotipos mais tradicionalmente consumidos entre as comunidades indígenas locais.
\end{abstract}

Palavras-chave: Capsicum, Roraima, pimenta, Amazônia

\section{Capsicum Peppers Cultivated in Roraima, Brazilian Amazonia. I. Domestic Species}

\begin{abstract}
Domesticated pepper species of the genus Capsicum cultivated in Roraima State, northern Brazilian Amazonia were surveyed. We collected 163 accessions of this group among small farmers (colonists) and indigenous communities. Capsicum chinense Jacq. (76.7\%) was the species with largest number of accessions, followed by $C$. frutescens L. (9.8\%), C. аппиит L. $(8.0 \%)$ and C. baccatum v. pendulum Wild. (5.5\%). Main fruit shapes were "long" (42.9\%) and "oval" $(27.0 \%)$. C. chinense presented the largest diversity in forms while the other species showed mainly the "long" shape. The predominant color of mature fruits was red $(64.4 \%)$. C. chinense presented a better distribution among the basic colors (yellow - 44.8\% and red - 55.2\%), without considering the different tonalities of individual accessions (orange, dark red etc). The sensorial pungency leves were "high" (62.6\%), followed by "medium" (16.0\%), "low" (15.3\%) and "very high" (6.1\%). Within the 105 accessions of red coloration $67.6 \%$ possessed "high" or "very high" pungency. C. chinense accessions popularly known as "murupi" and "olho-de-peixe", together with "malagueta" (C. frutescens), are the most traditional types consumed among local indige-nous communities.
\end{abstract}

Key-words: Capsicum, Roraima, pepper, Amazonia

Instituto Nacional de Pesquisas da Amazônia - INPA - Coordenação de Pesquisas em Ecologia - CPEC - R. Coronel Pinto 341

2Empresa Brasileira de Pesquisa Agropecuária - EMBRAPA - Roraima. Rodovia BR 174, km 8 - Distrito Industrial - Caixa Post

${ }^{3}$ Governo do Estado de Roraima - Secretaria de Planejamento (CTA-INPA/GERR) - R. Coronel Pinto 341 - Centro - Caixa Post

Museu Integrado de Roraima - MIRR - Av. Brigadeiro Eduardo Gomes sn - Parque Anauá 69301-010 Boa Vista/Roraima - Fone/fax: 0xx-95-623 1733 - Boa Vista - Roraima 


\section{Introdução}

Pimentas do gênero Capsicum (Solanaceae) vêm sendo utilizadas sob diferentes formas por uma ampla gama de culturas humanas ao redor do planeta. Este gênero pode ser associado, por exemplo, à medicina tradicional humana (Cichewicz \& Thorpe, 1996; Molina-Torres et ai, 1999; Otero et ai, 2000), ao combate de enfermidades em criações domésticas (Mtambo et ai, 1999), ou mesmo a ritos de passagem que destaquem a bravura de jovens guerreiros indígenas (obs. pessoal). Entretanto, o gênero Capsicum é mais fortemente relacionado a produtos condimentares que dão gosto e cheiro a diferentes pratos em variadas cozinhas mundiais. Isto é devido aos alcalóides (capsaicinóides) contidos em seus frutos que na maioria das vezes produzem uma sensação organoléptica de ardência (Bosland, 1996). Pimentas deste gênero também são uma excelente fone de $\beta$ caroteno, vitamina A e vitamina C (Vargas, 1985; Gbolade et ai, 1997; Mejía et ai., 1998). Além disto, os capsaicinóides dos frutos são caraterizados por possuírem uma alta atividade biológica nos campos da farmacologia, neurologia e alimentação (Perucka \& Oleszek, 2000).

Incontestavelmente exclusivo das Américas, o gênero Capsicum se expandiu com grande velocidade por outras partes do mundo a partir do século XVI, quando a movimentação das populações européias entre as

\section{comunidades} indígenas foi intensificada pelo maior nível de relacionamento entre estes dois povos (Heiser \& Smith, 1953; Casali \& Couto, 1984; Garcia, 1991). Vários registros arqueológicos indicaram que o gênero Capsicum já vinha sendo consumido há pelo menos 8.600-5.600 a.C. nas regiões andinas do Peru (Caverna Guitarrero) e 6.500-5.500 a.C. no México (Nuez et ai, 1998). Estes achados sugerem que, juntamente com os gêneros Phaseolus (feijão) e Curcubita (abóboras), as pimentas faziam parte das primeiras plantas a serem domesticadas nas Américas e, portanto, encontravam-se na maioria dos cardápios de uma grande parte das populações existentes à época.

Na Amazônia brasileira, embora o sistema regional de manejo integrado possa ser considerado fraco do ponto de vista da comercialização interna e externa, o cultivo de pimentas do gênero Capsicum pode ser uma importante fonte alternativa de geração de divisas para as populações agrícolas (indígena e nãoindígena) da região. Isto porque há um rico potencial regional muito pouco conhecido e/ou explorado de forma organizada, embora se admita que o Brasil e, principalmente a Amazônia, seja um importante centro secundário de espécies domesticadas (Reifschneider, 2000).

Em Roraima, poucas são as citações históricas sobre o uso de pimentas pelas populações tradicionais locais. Apenas na extensa investigação etnológica de Koch-Grünberg (197982), que percorreu toda a região 
dominada pelo Monte Roraima e Serra Parima entre os anos de 1911-13, foi possível encontrar vestígios do uso de plantas do gênero Capsicum entre as populações locais. Koch-Grünberg fez várias citações (Tomo I, pags. 43; 257 ; 275; 307) sobre o uso do "... caldo de pimienta ..." (provavelmente a damorida), da "... salsa de pimienta ..." (provavelmente um tipo de molho) e da "... pimienta fresca ..." (frutos frescos adicionados diretamente à alimentação), todos muito utilizados entre os Macuxi, Wapichana, Yekuana e por ele mesmo ao longo de suas caminhadas pelas áreas de mata e savanas regionais.

Mais recentemente, na segunda metade da década de 1960, Pereira (1980) apresentou uma imagem de vários indígenas saboreando a "... tamorida..." (damorida) como uma das formas de socialização realizada entre a etnia Ingarikó do alto rio Cotingo. Diniz (1972) também descreveu a "damorida" como uma das "... comidas típicas..." de maior aceitação entre o povo Macuxi sendo que o caldo é um preparado à base de "... pimentas e folhas de pimenteira..." e algum tipo de proteína animal (peixe ou carne de caça). Berg \& Silva (1988), em um levantamento sobre a flora medicinal de Roraima, indicaram que a pimenta malagueta $(\mathrm{C}$. frutescens L.) era utilizada no combate ao "pano branco" de pele. Na seqüência, investigações etnobotânicas de Azevedo Lima (1990) e Milliken et al. (1992) atestaram o plantio de pimentas do gênero Capsicum nas roças WaimiriAtroari (extremo sul de Roraima) para aproveitamento alimentar. Outro levantamento abordado por Milliken \& Albert (1997) e Milliken et al. (1999) também relatou a utilização de $\mathrm{C}$. frutescens L. como planta de uso medicinal (infecções respiratórias e oftalmia) pela etnia indígena Yanomami. A mesma espécie também era usada no passado para tratamento da malária por algumas comunidades Wapichana do leste de Roraima (Milliken, 1997).

Estes relatos não esgotam o vasto uso de Capsicum por todas as comunidades urbanas e rurais atualmente residentes em Roraima. Entretanto, devido a enorme movimentação de migrantes nas últimas décadas, uma parte dos morfotipos presentes hoje em Roraima não possui nem mesmo o nome comum regional, sendo que seus potenciais usos econômicos ao sistema de produção local ainda são pouco conhecidos. Esta mesma observação pode ser feita para as espécies e morfotipos regionais que transitam tradicionalmente pelas culturas humanas situadas geograficamente entre Roraima, o sul da Venezuela e o sul-sudoeste da Guiana. Este rico patrimônio genético, construído ao longo do tempo pelas comunidades indígenas regionais, pode estar sofrendo fortes modificações nas variedades tradicionais devido à bagagem de morfotipos introduzidos, em maior escala, pelo elevado fluxo de migrantes dos últimos anos. A coleta e a manutenção de pimentas são de grande importância porque abre espaço para um melhor conhecimento do potencial de adaptação e de resistências deste gênero (Bianchetti et al, 1999).

Este trabalho relata um 
levantamento das espécies e morfotipos de pimentas domesticadas do gênero Capsicum cultivadas em Roraima, realizado com o propósito de montar um banco gênico regional. A idéia é dar conhecimento básico deste rico potencial da flora local que é fortemente utilizado nesta região da Amazônia pelos segmentos urbano e rural.

\section{Material e Métodos}

\section{Caracterização da Área de Estudo}

Roraima possui $225.116 \mathrm{~km}^{2}$ de área e está situado no extremo norte da Amazônia brasileira. Sua estrutura fitofisionômica pode ser dividida, a grosso modo, em três grandes ambientes tomando como base o ano de 2000: (1) sistemas florestais (densos e não-densos), com aproximadamente $70 \%$ da área, (2) sistemas de vegetação aberta, caracterizados pelas savanas $(16 \%) \mathrm{e}$ campinas-campinaranas do interflúvio rio Branco-rio Negro (11\%) e (3) sistemas antrópicos representados pelas pastagens, capoeiras e cultivos agrícolas (3\%). Os dois primeiros blocos fisionômicos formam um intrincado mosaico de estruturas vegetacionais distribuídos em função do relevo, do solo e das condições climáticas a que estão afetos.

Três são as classificações climáticas regionais pelo sistema de Kõppen: (1) "Aw" - predomina nas áreas de savana do norte-nordeste e a pluviometria média anual varia de 1.100-1.700mm, (2) "Af - caracterizado pela dominância de sistemas florestais úmidos de baixa altitude da região sul, com pluviometria acima de $2.000 \mathrm{~mm}$ anuais e (3) "Am" - forma uma espécie de corredor intermediário entre as savanas e as florestas úmidas, com pluviometria variando de 1.700-2.000 $\mathrm{mm}$ a.a. (Barbosa, 1997). Em geral, embora haja variações microclimáticas entre os extremos norte e sul do estado, o período seco se estende de dezembro a março e, a fase úmida é mais pronunciada entre maio e agosto.

As regiões norte-nordeste e leste de Roraima são tradicionalmente habitadas pelas etnias indígenas Macuxi, Wapichana, Ingarikó, Taurepang e Patamona. A região noroeste e oeste é a área de ocupação das comunidades Yanomami e Yekuana. O centro-sul vem sendo ocupado por fazendas de gado de corte em sistema extensivo e por projetos de assentamento humano incentivados pelo poder público, principalmente a partir de meados da década de 1970. Por fim, nos extremos sul e sudeste estão situadas as etnias indígenas Waimiri-Atroari e Wai Wai, respectivamente.

\section{Locais de Amostragem}

Imaginando este mosaico de fisionomias étnicas e vegetais presentes em Roraima, a amostragem das espécies e morfotipos de pimentas regionaais transcorreu pelo estado, aproveitando contatos com (1) as Casas do Produtor Rural estabelecidas nas vilas e agrovilas formadas para atender a população migrante em áreas de floresta, (2) povoações e sedes de fazendas presentes nas áreas de savanas regionais, e (3) lideranças indígenas das etnias estabelecidas 
tradicionalmente em Roraima. Para tanto, foram estabelecidas 39 localidades centrais de amostragem ( 9 indígenas, 25 não-indígenas e 5 conjugadas) distribuídas por 14 dos 15 municípios existentes em Roraima entre maio/2000 e junho/2001 (Tab. 1). Estas localidades serviam como base de referência geográfica e ponto de partida para coletar os morfotipos e espécies de pimentas domesticadas que orbitavam em suas proximidades tanto na forma de hortas caseiras como em sistemas semi-comerciais.

\section{Inventário e Coleta do Material}

Cada espécie e/ou morfotipo encontrado em campo foi registrado com as seguintes informações: (a) nome científico e vulgar (quando possível), (b) localidade, juntamente com o nome da pessoa ou do órgão cedente, (c) coletor e data de coleta, (d) forma de uso do fruto pelo cedente, e (e) dimensões (transversal e longitudinal), peso, cor e nível de pungência (ardência) do fruto. No quisito pungência, o método adotado foi o sensorial, através das seguintes classes estimadas de ardência: (a) "muito alta" (MA) - acima de 150.000 SHU (Scoville Heat Unit - unidade utilizada para avaliar a pungência), (b) "alta" (A) - entre 75.000 e 150.000 SHU, (c) "média" (M) - entre 25.000 e 75.000 SHU, e (d) "baixa" (B) - inferior a 25.000 SHU. Os intervalos de classe são propositadamente largos devido aos erros que a análise sensorial naturalmente provoca. Eles foram baseados e modificados a partir da descrição apresentada em Reifschneider (2000). O critério de classificação das espécies foi baseado nas chaves dicotômicas de identificação que são apresentadas em DeWitt \& Bosland (1966) e Nuez et al.\{ 1998) e, nos aspectos morfológicos das flores descritos em Reifschneider (2000).

A cada coleta, em média 5-10 frutos maduros eram selecionados aleatoriamente do indivíduo (planta) observado e acondicionados em sacos de plástico para transporte até o laboratório para catalogação, fotografia e extração de sementes para reprodução na sede da EMBRAPA em Boa Vista, Roraima. Sempre que possível, foram tomadas imagens 3 fotográficas do local e do indivíduo vegetal investigado, além de coletada uma amostra fértil da planta para depósito no Herbário do Museu Integrado de Roraima e/ou remessa ao especialista quando existissem dúvidas quanto à identificação da espécie. Quando o indivíduo observado em campo estava sem floração (somente frutos), o material para herborização foi oriundo da reprodução do material genético coletado.

Todo o material reproduzido está servindo para a criação de um banco de sementes de Capsicum na EMBRAPA de Roraima. Além disto, parte dos frutos originariamente coletados estão sendo resguardados para análise do DNA e detecção das similaridades genéticas.

\section{Resultados e Discussão}

\section{Número de Espécies e Morfotipos}

Foram identificadas 4 espécies entre os 163 acessos pertencentes ao grupo das "domesticadas": $C$. chinense Jacq. (125 ou 76,7\%), C. frutescens $\mathrm{L}$. 
Tabela 1. Posição geográfica das localidades amostrais de referência para coleta de pimentas domesticadas do gênero Capsicum em Roraima, por município e grupo étnico social.

\begin{tabular}{|c|c|c|c|c|c|}
\hline Código & Localidade & Município & Longitude & Latitude & $\begin{array}{c}\text { Grupo Social Étnico } \\
\text { Predominante }\end{array}$ \\
\hline 1 & Alio Alegre & Alto Alegre & $61019^{\prime} 02 " \mathrm{~W}$ & $2059^{\prime} 15^{\prime \prime} \mathrm{N}$ & n-indígena \\
\hline 2 & Col. Alto Alegre (Vic. 4) & Alto Alegre & $61020^{\prime} 54 "$ W & $2058^{\prime} 54^{\prime \prime} \mathrm{N}$ & n-indígena \\
\hline 3 & Paredão (Vicinal 1) & Alto Alegre & $61023 " 57 "$ W & 30 OV $38 " \mathrm{~N}$ & n-indígena \\
\hline 4 & Amajari & Amajari & 610 22' 04" W & 30 $39 " 00 " \mathrm{~N}$ & n-indígena \\
\hline 5 & E. E. Ilha de Maracá & Amajari & 610 26' 54" W & 3o $21^{\prime} 49^{\prime \prime} \mathrm{N}$ & n-indígena \\
\hline 6 & Boa Vista & Boa Vista & $60041^{\prime} 26 " \mathrm{~W}$ & $2049^{\prime} 22 " \mathrm{~N}$ & n-indígena \\
\hline 7 & Igarapé do Carrapato & Boa Vista & $60038^{\prime} 12^{\prime \prime}$ W & $2054^{\prime} 16^{\prime \prime} \mathrm{N}$ & n-indígena \\
\hline 8 & Taculu & Bonfim & $59050^{\prime} 01 " \mathrm{~W}$ & $3021^{\prime} 01^{\prime \prime} \mathrm{N}$ & n-indígena \\
\hline 9 & P.A. Caxias (Vicinal 9) & Canta & & & n-indígena \\
\hline 10 & Petrolina do Norte & Caracaraí & $60059^{\prime} 29 " \mathrm{~W}$ & $1034^{\prime} 02 " \mathrm{~N}$ & n-indígena \\
\hline 11 & Entre Rios & Caroebe & 59025 '34" W & Oo 32' 11" N & n-indígena \\
\hline 12 & Iracema & Iracema & 60003 ' 12" W & $2009^{\prime} 57^{\prime \prime} \mathrm{N}$ & $\dot{\eta}$ ind ígena \\
\hline 13 & Vila São Raimundo (1) & Iracema & 60058 ' 23" W & $2015^{\prime} 46^{\prime \prime} \mathrm{N}$ & n-indígena \\
\hline 14 & Vila São Raimundo (2) & Iracema & 60058 ' $40^{\prime \prime}$ W & $2014^{\prime} 50^{\prime \prime} \mathrm{N}$ & fi ind ígena \\
\hline 15 & Mucajaí & Mucajaí & $60055^{\prime} 17 "$ W & 2026 '11" N & n-indígena \\
\hline 16 & Nova Esperança & Mucajaí & 61032 ' 33" W & $2043^{\prime} 22 " \mathrm{~N}$ & n-indígena \\
\hline 17 & Serra da Prata & Mucajaí & 61011 '01" W & $2019^{\prime} 01 " \mathrm{~N}$ & n-indígena \\
\hline 16 & Vila do Apiaú & Mucajaí & $61018 " 24 "$ W & $2033^{\prime} 17^{\prime \prime} \mathrm{N}$ & n-indígena \\
\hline 19 & Pacaraima & Pacaraima & 610 09'09" W & 4028 '38" N & fi ind ígena \\
\hline 20 & Rorainópolis (Vicinal 9) & Rorainópolis & 60025 '01" W & Oo 56'01" N & n-indígena \\
\hline 21 & Vila do Equador (Vic. 2) & Rorainópolis & $60029^{\prime} 36 " \mathrm{~W}$ & Oo 04' 22" N & n-indígena \\
\hline 22 & Vila do Juridiá & Rorainópolis & $60041^{\prime} 41^{\prime \prime} \mathrm{W}$ & Oo 12' $31 " \mathrm{~s}$ & n-indígena \\
\hline 23 & BR 174 (km 97) & Rorainópolis & 60040 '03" W & Oo 01' 23" s & n่ indígena \\
\hline 24 & BR-210 (Sítio Nunes) & S. L. Anauá & 60005 '00" w & 1002 ' $00 " \mathrm{~N}$ & n-indígena \\
\hline 25 & S. L. do Anauá (Vic. 18) & S. L. Anauá & 60002 '01" W & $1000^{\prime} 01 " \mathrm{~N}$ & fí-ind ígena \\
\hline 26 & Hakoma (Yanomami) & Alto Alegre & $63035^{\prime} 00^{\prime \prime}$ W & $2036^{\prime} 00^{\prime \prime} \mathrm{N}$ & indígena \\
\hline 27 & Milho (Wapichana) & Boa Vista & 60024 ' 45" W & 3o $23^{\prime} 16^{\prime \prime} \mathrm{N}$ & indígena \\
\hline 28 & Tarame (Macuxi) & Normandia & 60001 '05" W & $3050^{\prime} 11 " \mathrm{~N}$ & indígena \\
\hline 29 & Auaris (Mayongong) & Pacaraima & 640 29' $16 "$ W & $4000^{\prime} 06^{\prime \prime} \mathrm{N}$ & indígena \\
\hline 30 & Barro (Macuxi) & Pacaraima & 600 47' 50" W & $40{ }^{12}$ '.11" N & indígena \\
\hline 31 & Contão (Macuxi) & Pacaraima & $60032^{\prime} 40^{\prime \prime} \mathrm{W}$ & $4010 " 16 " \mathrm{~N}$ & indígena \\
\hline 32 & Maturuca (Macuxi) & Uiramuitã & $600.05^{\prime} 59^{\prime \prime} \mathrm{W}$ & $4027^{\prime} 55^{\prime \prime} \mathrm{N}$ & indígena \\
\hline 33 & Constantino (Macuxi) & Uiramutã & 600 22' 17" W & $4005 ' 52 " \mathrm{~N}$ & indígena \\
\hline 34 & Sand Hill (Patamona) & Uiramutã & $59059^{\prime} 14^{\prime \prime}$ W & 4058 '06" N & indígena \\
\hline 35 & Placa & Normandia & $6003 \mathrm{~V} 07 " \mathrm{~W}$ & $4010^{\prime} 41 " \mathrm{~N}$ & conjugada(1) \\
\hline 36 & Vila Surumú & Pacaraima & $60047^{\prime} 35^{\prime \prime}$ W & $4011^{\prime} 02 " \mathrm{~N}$ & conjugada \\
\hline 37 & Uiramutã & Uiramuitã & 600 09' $34 "$ w & $4035^{\prime} 45^{\prime \prime} \mathrm{N}$ & conjugada \\
\hline 38 & Vila do Soco & Uiramuitã & $60010^{\prime} 40^{\prime \prime} \mathrm{W}$ & $4028^{\prime} 10^{\prime \prime} \mathrm{N}$ & conjugada \\
\hline 39 & Normandia & Normandia & $59037^{\prime} 40 " \mathrm{~W}$ & 3052 ' $54 " \mathrm{~N}$ & conjugada \\
\hline
\end{tabular}

"'Conjugada = localidades com forte missigenação ou sem predomínio de etnias. 
$(9,8 \%)$, C. annuиm L. $(8,0 \%)$ e C. frutos maduros foi a vermelha (105 ou baccatum L. (5,5\%) (Tab. 2). 64,4\%). Isoladamente, C. chinense Apenas C. pubescens Ruiz \& Pavon não foi encontrada dentre as cinco espécies de pimentas domesticadas conhecidas (ver Heiser \& Pickersgill, 1969). A proporção acima relatada representa uma média aproximada de ocorrência e distribuição destas espécies por todas as localidades e municípios visitados em Roraima. As formas do fruto mais encontradas entre as domesticadas foram a "alongada" (70 ou $42,9 \%)$ e a "ovalada" (44 ou $27,0 \%)$. C. chinense foi a que apresentou a maior diversidade de formas enquanto que as outras espécies estavam mais concentradas na "alongada". A cor predominante dos obteve uma melhor distribuição entre as cores básicas (amarela - 44,8\% e vermelha - $55,2 \%$ ), independente das diferentes tonalidades assumidas por cada acesso (creme, alaranjado, vermelho-escuro etc). O nível de pungência (ardência) com maior número de registros foi o "alto" (102 ou 62,6\%), seguido do "médio" (16,0\%), "baixo" $(15,3 \%)$ e "muito alto" $(6,1 \%)$. Dos 105 acessos de cor vermelha, $71(67,6 \%)$ possuíam pungência "alta" ou "muito alta".

Restringindo-se apenas às diferenças morfo-colorimétricas do fruto (desconsiderando a pungência), foi constatado a presença de 78 morfotipos de pimentas domesticadas

Tabela 2. Espécies e acessos de pimentas do gênero Capsicum encontradas entre maio/2000 e junho/2001 em Roraima.

\begin{tabular}{|c|c|c|c|c|c|c|c|c|c|c|c|c|c|}
\hline \multirow[t]{2}{*}{ Espécies (1) } & \multirow[t]{2}{*}{ Acessos } & \multicolumn{6}{|c|}{ Formado Fruto } & \multicolumn{2}{|c|}{ Cor Predominante"' } & \multicolumn{4}{|c|}{ Teor de Pungência(3) } \\
\hline & & alongada & ovalada & redonda & campanulada & cônica & s.l. (4) & vermelha & amarela & MA & A & M & B \\
\hline C.annuum & 13 & 6 & 2 & 3 & 1 & 1 & & 1 & 2 & & 2 & 1 & 10 \\
\hline C . baccalum $\mathrm{v}$. pendulum & 9 & 4 & & & 5 & & & 9 & & & 2 & 6 & 1 \\
\hline C. chinense & 125 & 44 & 42 & 16 & 9 & 8 & 6 & 69 & 56 & 9 & 84 & 19 & 13 \\
\hline C. frutescens & 16 & 16 & & & & & & 16 & & 1 & 14 & & 1 \\
\hline Total Geral & 163 & 70 & 44 & 19 & 15 & 9 & 6 & 105 & 58 & 10 & 102 & 26 & 25 \\
\hline (\%) & & 42,9 & 27,0 & 11.7 & 9,2 & 5,5 & 3,7 & 64,4 & 35,6 & 6,1 & 62.6 & 16.0 & 15.3 \\
\hline \multicolumn{14}{|c|}{$\begin{array}{l}\text { (1) Outros } 17 \text { acessos foram encontrados ( } 6 \text { não-identificados, } 3 \text { silvestres e } 8 \text { semidomesticadas); } \\
\text { Para frutos maduros, incluindo os diferentes tons que ambas as cores podem assumir (vermelho: escuro, } \\
\quad \text { claro / amarelo: creme, alaranjado, etc); } \\
\text { (3) Nível de Pungência (Ardência) Sensorial: os valores abaixo indicados são estimativas em função de outros } \\
\text { estudos já realizados: } \\
\text { MA = Muito Alta : acima de } 150.000 \mathrm{SHU} \mathrm{(Scoville} \mathrm{Heat} \mathrm{Unit)} \\
\text { A = Alta : entre } 75.000 \text { e } 150.000 \mathrm{SHU} \\
\text { M = Média : } 25.000 \text { e } 75.000 \mathrm{SHU} \\
\text { B = Baixa : menos de } 25.000 \mathrm{SHU} \\
\text { (4) s.f. = sem forma definida. }\end{array}$} \\
\hline
\end{tabular}


transitando pelo lado brasileiro da fronteira entre Roraima, Venezuela e Guiana. Este valor é bem maior do que os 18 tipos de pimentas (C. chinensè) que eram cultivadas por comunidades indígenas na Amazônia colombiana ao final da década de 1980 (Garcia, 1991). Entretanto, ainda se encontra abaixo do real potencial genético local, visto que coleções como da EMBRAPA/ CENARGEN e da New Mexico State University possuem centenas de acessos registrados em seus bancos de dados.

\section{Espécies Domestícadas em Roraima}

C. baccatum é uma espécie originária dos Andes e provavelmente foi domesticada no Peru (DeWitt \& Bosland, 1993). Em Roraima, foram determinados apenas acessos da variedade $C$. baccatum v. pendulum Willd. Seus frutos são geralmente de comprimento médio $(4 \mathrm{~cm})$ para grande $(6-8 \mathrm{~cm})$ e suas flores são caracterizadas pela coloração branca com manchas esverdeadas ou marrons na base das pétalas. A distribuição dos morfotipos regionais se estabeleceu da seguinte forma: (1) chapéu-de-frade "de cheiro" (teor de pungência médio) e (2) dedode-moça "ardosa" (alto), "de cheiro" (médio) e "doce" (baixo) (Fig 1).

As pimentas chapéu-de-frade foram, muito provavelmente, introduzidas recentemente (últimos 20 anos) com a entrada de sementes selecionadas que são vendidas em casas especializadas locais e/ou devido ao fluxo migratório mais intenso registrado nas décadas de 1980 e 1990. Este grupo é encontrado tanto em assentamentos rurais do centro-sul do Estado como em aldeamentos indígenas do extremo nordeste. Embora este tipo de pimenta não tenha sofrido variações no formato geral, o teor de pungência já possui pequenas variações dentro de um único morfotipo. As pimentas dedo-de-moça sugerem que receberam o mesmo tratamento de introdução e dispersão local, mantendo a forma geral do fruto mas, variando os teores de pungência por algum fator não determinado. Em ambos os casos, chapéu-de-frade e dedo-de-moça, os supermercados de Boa Vista atribuem um valor médio de US\$ 3.40/kg do fruto "in natura" (US\$ $1.00=\mathrm{R} \$ 2,35-$ junho/2001). Em feiras livres e hortas do interior do Estado o preço é menor e muito variável, dependendo de negociação entre as partes. No caso específico da dedo-de-moça, uma parte da movimentação comercial é oriunda de hortas de Brasília devido à boa aceitação local.

As demais espécies \{annuumchinense-frutescens) são reconhecidamente relacionadas e formam um tripé ancestral mútuo, significando que possuem um mesmo "pool" genético (Eshbaugh, 1979; De Witt \& Bosland, 1996; Zewdie \& Bosland, 2001). As três espécies somam juntas mais de $94 \%$ de todos os morfotipos levantados em Roraima. Embora haja uma carga de polinização cruzada natural nos morfotipos deste tripé (seguida ou não de uma seleção antrópica local), cada uma das espécies possui sua característica individual. C. 
Figura 1. Morfotipo "dcdo-de-moça" (C. baccatum) coletado na sede do Município de Boa Vista, Roraima.

annuum (formas cultivadas do pimentão) possui flores brancas, robustas e, em geral, com um diâmetro entre 1,4-1,8 cm. Sua área de abrangência estava concentrada, à época do descobrimento, na região compreendida entre o sul dos Estados Unidos e o Panamá (Nuez et al, 1998).

Dos pimentões cultivados encontrados em Roraima, destacam-se a "pimenta americana" (vermelho, alongado, baixa pungência - 25,0 g/ fruto), o "pimentão bola" (Fig. 2) do tipo "Large Cherry" (vermelho, redondo, baixa pungência - 9,5 g/fruto) e o "pimentão indígena" que lembra muito a cultivar "California Wonder" (vermelho, redondo, baixa pungência - 36,0 g/fruto). Os dois primeiros pela raridade de serem encontrados em hortas caseiras locais e, o terceiro, pela seleção, de anos, criada por uma comunidade indígena Macuxi da região do médio rio Surumu (Aldeia do Barro). Os demais morfotipos de pimentões são provenientes de sementes vendidas nas casas especializadas e, são encontrados facilmente em quase todas as hortas e municípios de Roraima pela boa aceitação do mercado consumidor. Seu valor de mercado é, em média, de US\$ 1.00-1.20 / kg "in natura" (junho/2001) nas feiras livres e supermercados regionais.

C. frutescens é popularmente conhecido por pimenta malagueta. A flor de frutescens é quase sempre verde-clara, podendo variar a coloração para branco-esverdeada. Sua corola não possui manchas e os 
Figura 2. Morfotipo "pimentão bola" (C. anmium) coletado na sede do Município de Alto Alegre, Roraima.

lóbulos se posicionam para trás após a antese. Seu centro de ocorrência à época da colonização européia se estendia por quase todo o Brasil, além dos demais países tropicais e subtropicais da América (Martin et al, 1979; Nuezeítf/., 1998).

Neste levantamento, esta espécie contribuiu com 16 acessos distribuídos em dois grupos: (a) malagueta "ardosa" (vermelha, alto teor de pungência - frutos graúdos ou pequenos) e (b) malagueta "doce" (vermelha, baixo teor - fruto pequeno). O primeiro grupo foi observado em todos os municípios de Roraima (Fig. 3). É o grupo de melhor distribuição entre as populações humanas locais e a de melhor aceitação no mercado re- gional. Além disto, é o que as comunidades indígenas mais conhecem e consomem, incluindo-as em alguns ritos culturais tradicionais das aldeias. Seu preço "in natura" atinge o valor de US\$ 8.20-10.40 / kg nos supermercados de Boa Vista (junho/2001) porque, além da natural dificuldade de coleta, na maioria das vezes falta produção regional para atender ao mercado consumidor. $\mathrm{O}$ segundo grupo (malagueta doce) foi detectado apenas em um município e, provavelmente, foi proveniente de uma polinização cruzada. Este morfotipo era utilizado pelo colono cedente como uma forma de adorno na alimentação devido ao seu baixo teor de pungência e, como defumação 
Figura 3. Pimenta "malagueta" (C. frutescens) coletada na Vila Jundiá, Município de Rorainópolis, Roraima.

(fruto seco queimado) para uso em rituais religiosos caseiros.

C. chinense é conhecida por ser a espécie de maior diversidade na bacia Amazônica (Casali \& Couto, 1984), podendo-se chegar a conclusão de que foi domesticada pelos indígenas da região e que, portanto,"... trata-se da mais brasileira dentre a espécies domesticadas" (Reifschneider, 2000). Isto foi francamente atestado pela grande quantidade de morfotipos encontrados em Roraima (> 76\% dos acessos coletados). C. chinense é uma espécie que se apresenta com duas'a cinco flores por nó e, o pedicelo, se encontra geralmente na forma pendente (Smith $\&$ Heiser, 1957). A corola pode variar da cor branca até a branco-esverdeada. As pétalas não possuem pontos ou marcas. Dos morfotipos encontrados em Roraima, os de maior distribuição são, sem dúvida, as pimentas "murupi" (23 dc pungência alta, 2 muito alta c
1 média), "de cheiro" (16 média e 7 baixa), "olho-dc-peixe" (14 alta), "murici" (6 alta) e "doce" (4 baixa).

A pimenta "murici" foi encontrada apenas em assentamentos humanos dirigidos (não-indígenas), sendo que, em dois casos, a origem das sementes foi relatada como vindo do Paraná c do Mato Grosso no final da década dc 1990. A coloração destas pimentas varia sempre dentro da tonalidade alaranjada. As pimentas "doce" também foram todas originárias das áreas de colonização agrícola, sendo pouco provável que tenham se originado das aldeias indígenas. A tonalidade predominante neste tipo foi a vermelha. A movimentação destes morfotipos (murici e doce) nesta região de fronteira foi mais acelerada a partir do "boom" garimpeiro do final da década de 1980 na Terra Indígena Yanomami, sendo difícil identificar a correta procedência destas variedades. 
Entretanto, "murupi" e "olho-depeixe" (Fig. 4) são, logo abaixo de "malagueta", as pimentas de maior consumo entre as comunidades indígenas devido ao alto grau de pungência que a maioria destes morfotipos apresenta. Isto vai ao encontro da afirmação de Nuez et al. (1998) de que as populações précolombianas possuíam muito pouca atração pelos tipos de baixa pungência. Tradicionalmente, estes povos selecionavam cultivares com alto nível de capsaicinóides. Apenas mais tarde foi que os tipos de baixo teor de pungência foram tomando maior importância (Heiser, 1976).

No caso de Roraima, as pimentas tipicamente indígenas são originariamente utilizadas de forma amassada nos alimentos ou na "damorida", um preparado à base de água, proteína animal (carne de caça ou pesca) e pimenta que é levado ao fogo e ingerido, em geral, com o beijú de mandioca (Manihot esculenta Crantz).
Outras formas encontradas atualmente são os molhos à base de soro de leite, tucupi (escorrido da mandioca) ou vinagre; todos também encontrados facilmente nos centros urbanos (Nascimento-Filho et al., sd). Além disto, quando existe excedente de produção, as comunidades preservam parte dela secando-a ao sol e, em seguida, moendo-a até se obter um pó fino (misturado ou não com sal) denominado de "jiquitaia". Este preparado é muitas vezes comercializado (quase sempre trocado) entre comunidades vizinhas ou mesmo dentro da própria comunidade no intuito de se obter outros produtos alimentares.

As pimentas denominadas como "de cheiro" (média e baixa pungência), são as que apresentam o maior potencial comercial atual entre todos os morfotipos locais. Vários colonos do centro-sul do estado possuem plantios de 2.000 a 5.000 pés. A produção é alta mas o preço de venda ao intermediário é baixo, atingindo

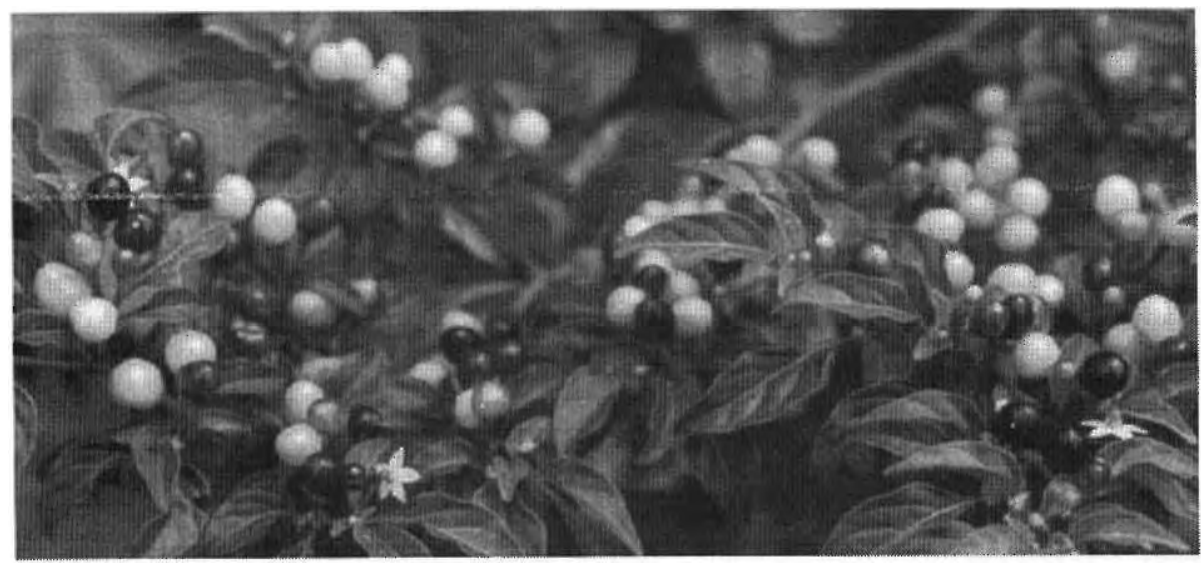

Figura 4. Pimenta "olho-de-peixe" (C. chinense) coletada nas proximidades da sede do Município de Pacaraima, Roraima. 
apenas US\$ 8.70 a US\$ 10.80 o saco de $20 \mathrm{~kg}$ "in natura", exclusivamente dentro do grau de maturação "verde". Além disto, o uso de defensivos contra pragas nas pimentas regionais (principalmente pulgões e cochonilhas) é comum e, tradicionalmente, despreza-se o período de carência para a coleta dos frutos. A maioria da produção desta região é enviada para o mercado consumidor de Manaus. No cinturão verde de Boa Vista também existe uma grande produção deste morfotipo que é direcionado para o mercado urbano local. Nas feiras livres o valor do produto é muito variável, entretanto, nos supermercados o preço de venda ao consumidor final varia de US\$ 1.10 a US\$ $1.50 / \mathrm{kg}$ "in natura" (junho/2001).

\section{Outras Espécies}

Além das espécies domesticadas que foram alvo deste estudo, foram observados outros 17 acessos de pimentas que foram enquadrados como silvestres (3), semidomesticadas (8) ou morfotipos não-identificados (6). Embora estes grupos estejam fora do objetivo central deste estudo, fazendo parte de um outro levantamento (Barbosa et ai, sd), é importante citar seu grande interesse genético e a forma que algumas comunidades indígenas e/ ou áreas de colonização delas se utilizam. Isto porque, apesar do grande papel que as pimentas domesticadas possuem no comércio mundial, é crescente o interesse pelos "alimentos étnicos" nos mercados dos países desenvolvidos (Pickersgill, 1997).

Este grupo apresenta, por exemplo, morfotipos da semidomesticada $C$. апnиит v. glabriusculum que possui variações na forma do fruto e na coloração floral. Também foi possível detectar três acessos de um morfotipo silvestre (C. $c f$ chinense), que habita de forma natural tanto a região do médio e alto rio Cotingo (> 200-250m de altitude), dentro da Área Indígena Raposa-Serra do Sol, quando alguns complexos rochosos próximos de Boa Vista e Mucajaí. $\mathrm{O}$ acesso indígena é conhecido por "pimi'ro" ("pimenta" na língua Macuxi) ou "Pimenta do Curupira" na linguagem usual e, chamou a atenção pelo freqüente uso dado pelas etnias regionais do nordeste do Estado. E uma espécie de pequeno porte que se dispersa naturalmente em áreas de agricultura em descanso sempre ao início do período chuvoso.

\section{Conclusões}

Pimentas domesticadas do gênero Capsicum cultivadas em Roraima possuem uma enorme diversidade de formas, cor e pungência. Embora com uma grande carga de morfotipos sendo introduzidos mais recentemente nesta região da Amazônia, ainda é possível identificar cultivares tradicionalmente cultivados pelos povos locais. Morfotipos como "malagueta" (C. frutescens), "murupi" (C. chinense) e "olho-de-peixe" (C. chinense) são os que circulam com maior intensidade e volume por entre as comunidades indígenas locais. As pimentas "de cheiro" (C. chinense) possuem maior circulação e movimento comercial entre as comunidades assentadas em vilas agrícolas do centro-sul de Roraima. Entretanto, espécies e morfotipos de menor conhecimento 
atual podem ter grande aproveitamento comercial devido às formas e sabores diferenciados daqueles usualmente encontrados no mercado local. Além disto, há um rico potencial ecológico depositado nas pimentas semidomesticadas e silvestres amazônicas. Estas necessitam de um estudo específico no sentido de determinação de seu potencial genético de utilização como referência de cruzamento na transferência de qualidades que interessem ao sistema de produção local, como por exemplo resistência e sabor.

\section{Agradecimentos}

O Governo de Roraima financiou este estudo através do $3^{\circ}$ Termo Aditivo (Sub-Projeto 1) do Convênio de Cooperação Técnico-Científica (027/99) - INPA/GERR. Os agricultores das regiões centro-sul do Estado, as lideranças indígenas e habitantes da cidade de Boa Vista, nos acolheram e gentilmente cederam espaço para que fossem feitas coletas em seus plantios caseiros ou semi-comerciais. Hiroshi Noda (INPA - Manaus) deu os primeiros incentivos e cedeu parte da literatura utilizada como referência neste artigo. Luciano Bianchetti (EMBRAPA/ CENARGEN) colaborou decisivamente na identificação de parte do material herborizado. Dr Warwick E. Kerr foi um forte incentivador deste estudo. Dois revisores anônimos contribuíram para a construção do texto final deste trabalho.

\section{Bibliografia citada}

Azevedo Lima, R.R.O. 1990. Levantamentos preliminares sobre as roças WaimiriAtroari. Relatório nào-publicado do Programa Waimiri Atroari / Convênio
FUNAI-ELETRONORTE, Manaus. 7p.

Barbosa, R.I. 1997. Distribuição das chuvas em Roraima. In: Barbosa, R.I.; Ferreira, E.; Castellón, E. (Eds.) Homem, Ambiente e Ecologia no Estado de Roraima. INPA, Manaus, p. 325-335.

Barbosa, R.I.; Luz, F.J.F.; Nascimento-Filho, H.R.; Maduro, C.B. sd. Pimentas do gênero Capsicum cultivadas em Roraima, Amazônia brasileira. Ill . Espécies semidomesticadas $\quad e \quad$ silvestres. (Manuscrito).

Berg, E.M. van den; Silva,M.H.L. 1988. Contribuição ao conhecimento da flora medicinal de Roraima. Acta Amazônica, 18(1/2):23-35.

Bianchetti, L.B.; Bustamante, P.G.; Reifschneider, F.J.; Silva, G.P. 1999. Coleta de germoplasma de espécies silvestres de Capsicum no Sudeste brasileiro. In: Anais do II Simpósio de Recursos Genéticos para América Latina e Caribe (SIRGEALC), 21 a 26/11/1999, EMBRAPA-CENARGEN, Brasília/DF. (CD-ROM).

Bosland, P.W. 1996. Capsicums: innovative use of an ancient crop. In: Janick, J. (Ed.) Progress in New Crops. ASHS Press, Arlington, p. 479-487.

Casali, V.W.D.; Couto,F.A.A. 1984. Origem e botânica dc Capsicum. Informe Agropecuário (Belo Horizonte), 10(113):8-10.

Cichewicz, R.H.; Thorpe, PA. 1996. The antimicrobial properties of chile peppers (Capsicum species) and their uses in Mayan medicine. Journal of Ethnopharmacology, 52:61-70.

DeWitt, D.; Bosland, P.W. 1993. The Pepper Garden - From the Sweetest Bell to the Hottest Habanero. Ten Speed Press, Berkeley, California. 240p.

DeWitt, D.; Bosland, P.W. 1996. Peppers of the World - An Identification Guide. Ten Speed Press, Berkeley, California. 219p.

Diniz, E.S. 1972. Os índios Makuxi de Roraima. Faculdade de Filosofia, Ciências e Letras de Marília, São Paulo. Coleção Teses n" 9 (Tese de Doutorado). 181 p. 
Eshbaugh, W.H. 1979. Biosystematics and evolutionary study of the Capsicum pubescens complex. In: National Geographic Society Research Reports (1970 projects), Nat. Geog. Soc, Washington DC, p. 143-162.

Garcia, A.J.V. 1991. El Aji (Capsicum chinense Jacq.), patrimônio cultural y fílogenético de las culturas Amazônicas. Colombia Amazônica, 5(1):161-185.

Gbolade, A.A.; Omobuwajo, O.R., Soremekun, R.O. 1997. Evaluation of the quality of Nigerian chillies for pharmaceutical formulations. Journal of Pharmaceutical and Biomedical Analysis, 15:545-548.

Heiser, C.B. 1976. Peppers - Capsicum (Solanaceac). In: Simmonds, N.W. (ed.) Evolution of Crop Plants. Longman, London, p. 265-268.

Heiser, C.B.; Smith, P.G. 1953. The cultivated Capsicum peppers. Economic Botany 7:214-227.

Heiser, C.B.; Pickersgill, B. 1969. Names for the cultivated Capsicum species (Solanaceae). Taxon, 18: 277-283.

Koch-Grünberg, T. 1979 82. Del Roraima al Orinoco (Tomo I, II e III). Ediciones del Banco Central de Venezuela, Caracas.

Martin, F.W.; Santiago, J.; Cook, A.A. 1979. Vegetablesfor the hot, humid tropics (Part 7. The Peppers, Capsicum Series). Science and Education Administration /U. S. Department of Agriculture, N ew Orleans. $18 \mathrm{p}$.

Mejia, E.G.; Quintanar-Hernández, J.A.; Loarca Pina, G. 1998. Antimutagenic activity of carotenoids in green peppers against some nitroarenes. Mutation Research, 416:11 19 .

Milliken, W. 1997. Traditional anti malarial medicine in Roraima, Brazil. Economic Botany, 51(3):212 237.

Milliken, W.; Albert, B. 1997. The use of medicinal plants by the Yanomami Indians of Brazil, Part II. Economic Botany, 51(3):264 278 .

Milliken, W.; Miller, R.P.; Pollard, S.R.; Wandelli, E.V. 1992. Ethnobotany of the Waimiri A troar $i$ Indians of Brazil. Royal
Botanic Gardens, Kew, London. 146p.

Milliken, W.; Albert, B.; Goodwin Gomez, G. 1999. Yanomami: a forest people. Royal Botanic Gardens, Kew, London. 161 p.

Molina Torres, J.; Garcia-Chávez, A.; Ramirez Chávcz, E. 1999. Antimicrobial properties of alkamides present in flavouring plants traditionally used in Mesoamarica: affinin and capsaicin. Journal of Ethnophamacology, 64:241 248.

Mtambo, M.M.A.; Mushi, E.J.; Kinabo, L.D.B.; Maeda Machang=u, A.; Mwamengele, G.L.M.; Yongolo, M.G.S.; Temu, R.P.C. 1999. Evaluation of the efficiency of the crude extracts of Capsicum frutescens, Citrus limon and Opuntia vulgaris against $\mathrm{Newcastle}$ disease in domestic fowl in Tanzania. Journal of Ethnopharmacology, 68:55 61 .

Nascimento Filho, H.R.; Barbosa, R.I.; Luz, F.J.F.; Maduro,C.B. sd. Pimentas do gênero Capsicum cultivadas em Roraima, Amazônia brasileira. II. Formas de Uso. (Manuscrito).

Nuez, F; Díez, M.J.; Ruiz, J.J.; Fe ${ }^{\wedge} \mathrm{Me}^{\wedge} \mathrm{dt}$ Cordova, P.; Costa, J.; Cataiá, M.S.; Gonzalez; J.A.; Rodríguez,A. 1998. Catálogo de semilias de pimien to. Ministério de Agricultura, Pesca y Alimentación / Instituto Nacional dc Invcstigación y Tecnologia Agraria y Alimentaria. Madrid. 108p.

Otero, R.; Nunez, V.; Barona, J. Fonnegra, R.; Jimenez, S.L.; Osório, R.G.; Saldarriaga, M.; Diaz, A. 2000. Snakebites and ethnobotany in the northwest region of Colombia. III. Neutralization of the hemorrhagic effect of Bothrops atrox venom. Journal of Ethnopharmacology, 73(1-2):233-241.

Pereira, N. 1980. Moronguêtá: Um Decameron Indígena (vol. I). Civilização Brasileira, Rio de Janeiro. 434p. ( I ed. em 1967).

Perucka, I.; Oleszek, W. 2000. Extraction and determination of capsaicinoids in fruit of hot pepper Capsicum annuum L. by spectrophotometry and high-performance liquid chromatography. Food Chemistry, 71:287-291. 
Pickersgill, B. 1997. Genetic resources and breeding of Capsicum spp. Euphytica, 96:129 133 .

Reifschneider, RJ.B. (org) 2000. Capsicum: pimentas e pimentões no Brasil. EMBRAPA-Hortaliças, Brasília. 113p.

Smith, P.G.; Heiser, C.B. 1957. Taxonomy of Capsicum sinense Jacq. and the geographic distribuition of the cultivated Capsicum species. Bulletin of the Torrey Botanical Club, 84(6):413-420.

Vargas, M. 1985. Recursos genéticos del chile picante: generalidades sobre su cultivo, evaluación y perpectivas futuras. CA TIE Actividades en Turrialba, 3(3): 4-7.

Zewdie, Y.; Bosland, P.W. 2001. Capsaicinoid profiles are not good chemotaxonomic indicators for Capsicum species. Biochemical Systematics and Ecology, 29:161 -169.

\section{Aceito para publicação em 22/05/2002}

